\title{
Special Issue on Efficiency in Wireless Software-Defined Radio Systems
}

\author{
Gerd Ascheid • Marc Adrat
}

Published online: 27 August 2013

(C) Springer Science+Business Media New York 2013

Efficient and effective broadband wireless technologies are required to support the foreseeable need to process and exchange large amounts of data. In this Special Issue of the Springer Journal of Signal Processing Systems various disciplines of such efficiency and effectiveness in the context of Software-Defined Radio (SDR) technologies are discussed.

At first, a selection of articles focuses on "spectral efficiency". Since frequency spectrum is known to be scarce and limited, it becomes a resource of increasing value whose utilization must be handled with care. The rapidly growing demand for higher data throughputs in wireless communication does not allow inefficient use of spectrum anymore. Two promising approaches to tackle the "spectral efficiency" issue are Cognitive Radio (CR) and Dynamic Spectrum Access (DSA). A Cognitive Radio is capable of learning and adapting to its transmission environment. Taking e.g. spectrum sensing results into account, the decisions for accessing the scarce frequency spectrum in the most flexible and effective manner are made. CRs are typically based on SDR technology.

Thus, the first set of articles puts emphasis on topics in the areas of Cognitive Radio, Cognitive Radio Networks, and Spectrum Sensing.

The second selection of articles addresses the problems of "energy efficiency" and "implementation efficiency". Especially in wireless communications these types of efficiencies are highly important to increase battery time of modern SDRbased platforms. The architectures of such SDRs are typically based on heterogeneous multi-processor hardware platforms

G. Ascheid

Chair for Integrated Signal Processing Systems RWTH, Aachen

University, Templergraben 55, 52056 Aachen, Germany

e-mail: gerd.ascheid@iss.rwth-aachen.de

M. Adrat $(\square)$

Fraunhofer FKIE, Fraunhoferstr. 20, 53343 Wachtberg, Germany

e-mail: marc.adrat@fkie.fraunhofer.de hosting General Purpose Processors (GPP), Digital Signal Processors (DSP), Field Programmable Gate Arrays (FPGA) and sometimes even more specific processing elements. Each type of processor has a specific advantage in one or the other performance criterion like energy consumption, flexibility, processing time, throughput, waveform portability, components reusability etc. Whenever a new waveform shall be implemented on an SDR, the system designer has to take the decision for the best possible trade-off between all of these, partly conflicting, criteria under the given circumstances. For instance, the portability of waveform components can be improved by fulfilling the constraints of the Software Communications Architecture (SCA) specification and by using common Application Programming Interfaces (API).

Thus, the second set of articles puts particular emphasis on topics in the areas of SDR and SCA Implementations.

\section{Cognitive Radio, Cognitive Radio Networks, and Spectrum Sensing}

The first five articles of this Special Issue fall into this area.

The first article "Cognitive Radio - A Current Snapshot and Some Thoughts on Commercialization for Future Cellular Systems" (10.1007/s11265-013-0837-0), written by Thomas Kaiser, Hanwen Cao, Wei Jiang, Feng Zheng sets the stage by providing a survey of the state of the art in cognitive radio (CR) technologies, the relevant stakeholders, CR regulations and standardization activities as well as current $\mathrm{CR}$ products, services and prototypes. The relevance of all these aspects for the commercialization of CR technologies in future cellular networks with special focus on LTE (Long Term Evolution) is assessed.

In the article "Mapping Cognitive Radio System Scenarios into the TVWS Context" (10.1007/s11265-013-0761-3) by Per H. Lehne, Ole Grøndalen, Richard MacKenzie, Dominique 
Noguet, and Vincent Berg, it is analyzed whether scenarios from the QoSMOS project (Quality of Service and MObility driven cognitive radio Systems) can be used within TV whitespace. Range estimations and capacity considerations are also presented. The article contains detailed information about the QoSMOS background, further research on cognitive femtocells and range estimation based on ITU recommendations.

"On Detecting Spectrum Opportunities for Cognitive Vehicular Networks in the TV White Space" (10.1007/s11265013-0765-z) by Haris Kremo and Onur Altintas deals with the usage of cognitive networks for Intelligent Transportation Systems in order to solve the growing spectrum demand due to emerging vehicular applications. Different aspects are analyzed like the usage of TV white spaces, the availability of geo-location information even in difficult situations (e.g. in a tunnel), and the utilization of diversity. The influence on the MAC (Medium Access Control) layer is investigated along with a discussion on standardization and regulatory issues. The article also contains research on database access latency versus mobility and presents an overview about the additional number of nodes needed to achieve fusion of sensing information in collaborative systems.

"A Novel Mitigation Scheme for JTIDS Impulsive Interference on LDACS System Based on Sensing and Symbol Retransmission" (10.1007/s11265-013-0763-1) is presented by Giulio Bartoli, Romano Fantacci, Dania Marabissi, Luigia Micciullo, Claudio Armani, and Roberto Merlo. The novel idea is to retransmit OFDM Symbols (Orthogonal Frequency Division Multiplex) to cope with distortions being introduced by legacy waveforms in the L-Band, specifically JTIDS (Joint Tactical Information Distribution System). Blanking of distorted time samples is used, which is suggested for instance in the LDACS-1 proposal (L-band Digital Aeronautical Communication System). In order to identify distorted time samples, a sort of energy detection is realized, and then the samples are blanked to zero before the Discrete Fourier Transform is calculated for demodulation. A new technique called Partial Combining is also introduced.

The article "A case-study of Simulation and Performance Evaluation of a SDR baseband Architecture" (10.1007/ s11265-013-0764-0) by Anthony Barreteau, Sébastien Le Nours, and Olivier Pasquier helps to assist system architects in the process of evaluating performance of potential architectures and of exploring the design space. The article presents a modeling approach and technique for improvement of the simulation speed of architecture models using a case study. The case study is related to the modeling of an adaptive multi-application and multi standard radio communication system architecture. Simulation results of the case study are provided aiming at a good compromise between simulation speed of architecture models and accuracy of provided estimations.

\section{SDR and SCA Implementations}

The other four articles are from this area.

The work on "Power efficient SDR Implementation of IEEE 802.11a/p Physical Layer" (10.1007/s11265-013-0773-z) by Daniele Lo Iacono and Teo Cupaiuolo is motivated by the need for future vehicle to vehicle $(\mathrm{V} 2 \mathrm{~V})$ and vehicle to infrastructure (V2I) communication in the automotive sector. A new Block Processor Engine (BPE) is proposed, running a Physical Layer which is based on the IEEE $802.11 \mathrm{a} / \mathrm{p}$ standard. The modifications required to adapt the standard for V2V and V2I communication are also described. Some results on speed-up options are discussed showing the effectiveness of multithreading in the BPE. In addition, the article describes the analysis of the power consumption of the BPE. The analysis is done based on a low-leakage $65 \mathrm{~nm}$ CMOS technology library and shows the results for the power consumption of the BPE architectural blocks for both standards. Several other techniques for reducing power consumption are presented.

The article "Numerical Aspects of MIMO OFDM PHY Layer Applications on SDR Platforms" (10.1007/s11265013-0766-y) written by D. Guenther, T. Kempf, and G. Ascheid discusses MIMO OFDM receiver algorithms (Multiple Input Multiple Output) for achieving bit error rates close to floating point performance while using limited fixedpoint precision. The different variations of QR-decomposition are compared and benchmarked with a MIMO-test system based on a multi-core platform. The use of individual fixedpoint formats for each functional block of MIMO preprocessing is also discussed in the article. Information about numerical precision requirements is given and results for the execution time (scalar operation count) for the QR-decomposition are presented.

In "Physical Layer Development Framework for OsmocomBB" (10.1007/s11265-013-0762-2) by Harald Kröll, Stefan Zwicky, Benjamin Weber, Christian Benkeser, and Qiuting Huang, a new environment is proposed which allows to do research on various layers of the GSM (Global System for Mobile Communication) protocol stack in a closed system. The Data Link and Network Layer implementations are based on an Open Source Mobile Communication Baseband (OsmocomBB) project and extended by Physical Layer aspects. In order to ensure real-time implementation, an 
embedded system is prototyped where critical parts are running on an FPGA. The article provides a detailed overview of the proposed framework for physical layer development in conjunction with OsmocomBB. New is also an embedded implementation of the main block of the framework which was previously executed in Matlab.

The article "A Methodology for Design of Scalable Architectures in Software Radio Networks: a Unified Device and Network Perspective" (10.1007/s11265-013-0767-x) by Maurizio Colizza, Marco Faccio, Claudia Rinaldi, and Fortunato Santucci introduces a novel approach called the Tissue Methodology and the development of a design pattern called the Tissue Pattern based on it. It can be used to model a protocol stack in the direction of a MANET (Mobile Ad-hoc NETwork). It aims at reducing the development time through the use of automatic code generation techniques. Further, the feasibility of the proposed method in the article was verified by implementing the Tissue Pattern model for IEEE 802.15.4 PHY Layer. Also, the related simulations results are presented. A detailed description of the SCA as a relating technology is also introduced.

We hope that this Special Issue at hand would prove to be helpful and informative for your reading.

Have fun reading the articles!

Acknowledgements In this Special Issue of the Springer Journal of Signal Processing Systems various disciplines of efficiency are discussed which are topics of key interest when designing wireless communication schemes.

As Guest Editors, we express our sincere gratitude and warm thanks to all the reviewers who devoted their time and effort to provide valuable feedback for this Special Issue. Their professionalism and support for both, authors and the Editorial Board, are much appreciated. Last but not least, we also appreciate the work and support of the Editors-in-Chief of the Journal of Signal Processing and the publishing team at Springer for setting up this Special Issue.

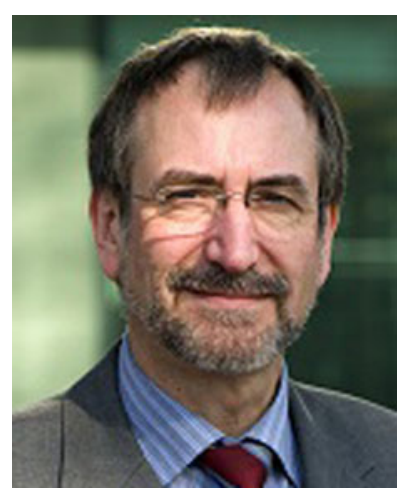

Gerd Ascheid received his Diploma and Ph.D. degrees in Electrical Engineering (Communications Eng.) from RWTH Aachen University. In 1988 he started as a co-founder CADIS GmbH which successfully brought the system simulation tool COSSAP to the market. From 1994-2003 Gerd Ascheid was Director / Senior Director with Synopsys, a California-based EDA market leader. In 2002 he was co-founder of LisaTek whose processor design tools are now part of the Synopsys product portfolio. Since April 2003 Gerd Ascheid heads the Institute for Integrated Signal Processing Systems of RWTH Aachen University. He is also coordinator of the UMIC (Ultra-high speed Mobile Information and Communication) Research Centre at RWTH Aachen University. His research interest is in wireless communication algorithms and application specific integrated platforms, in particular, for mobile terminals.

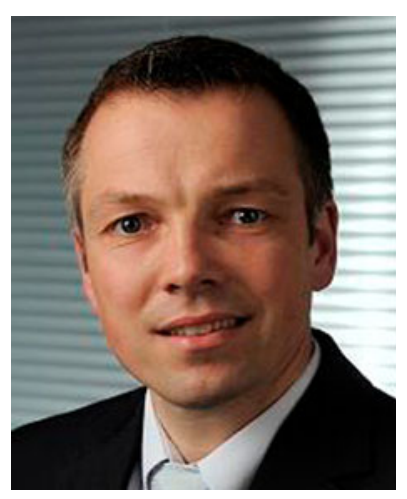

Marc Adrat received his Dipl.-Ing. degree in electrical engineering and the Dr.-Ing. degree (PhD) from RWTH Aachen University, Germany, in 1997 and 2003, respectively. Since April 2005, he is with the Fraunhofer Institute for Communication, Information Processing and Ergonomics (FKIE). He is the project manager for the Software Defined Radio activities in the Communication System department. His current research interests include software defined radio, cognitive radio, (military) waveform design as well as concepts for waveform development environments. Matters of particular interest are wideband networking waveforms as well as waveform development environments for portable, interoperable and energy-efficient waveforms. 